\title{
Schedules for Pneumococcal Vaccination of Preterm Infants: An RCT
}

\section{Short title: Schedules for Pneumococcal Vaccination of Preterms}

\section{Authors:}

Kent, Alison MBChB MRCPCH (1)

Ladhani, Shamez N MBBS PhD(2)

Andrews, Nick J PhD (3)

Scorrer, Tim MBChB MRCPCH (4)

Pollard, Andrew J MBBS PhD(5)

Clarke, Paul MD FRCPCH MRCP (6)

Hughes, Stephen M MBChB PhD (7)

Heal, Carrie MBChB MRCPCH (8)

Menson, Esse MBChB DPhil (9)

Chang, John MBChB FRCPCH (10)

Satodia, Prakash MBBS FRCPCH (11)

Collinson, Andrew C MBChB MD (12)

Faust, Saul N MBBS PhD (13)

Goldblatt, David MBChB PhD(14)

Miller, Elizabeth (15)

Heath, Paul T MBBS FRCPCH (1)

On behalf of the PUNS study group (see acknowledgements)

\section{Author Affiliations:}

1. Paediatric Infectious Diseases Research Group and Vaccine Institute, St George's, University of London, London, UK

2. Immunization, Hepatitis and Blood Safety Department, Public Health England, Colindale, London, UK

3. Statistics, Modelling and Economics Department, Public Health England, Colindale, London, UK

4. Neonatal Unit, Queen Alexandra Hospital, Portsmouth, UK

5. Oxford Vaccine Group, University of Oxford, and the NIHR Oxford Biomedical Research Centre, Oxford UK

6. Neonatal Unit, Norfolk and Norwich University Hospitals NHS Foundation Trust, Norwich, UK

7. Department of Immunology, Royal Manchester Children's Hospital, Manchester, UK

8. Neonatal Unit, Stepping Hill Hospital, Stockport, UK

9. Department of Paediatric Infectious Diseases, Evelina London Children's Hospital, London, UK

10. Neonatal Unit, Croydon University Hospital, London, UK

11. Neonatal Unit, University Hospital Coventry and Warwickshire NHS Trust, Coventry, UK

12. Neonatal Unit, Royal Cornwall Hospital, Truro, UK

13. NIHR Welcome Trust Clinical Research Facility, University of Southampton and University Hospital Southampton NHS Foundation Trust, Southampton, UK

14. Institute of Child Health, UCL, London, UK

15. Immunization, Hepatitis and Blood Safety Department, Public Health England, Colindale, London, UK 


\section{Corresponding author:}

A Kent, Paediatric Infectious Diseases Research Group and Vaccine Institute, St George's, University of London, London, UK. alisonkent@doctors.org.uk. Tel: 0044208725 5382, Fax: 00442087250170

Clinical trial registration: EudraCT number 2007-007535-23

\section{Funding statement}

This work was supported by Pfizer Ltd as an investigator-led study. The funder had no input into the conduct of the trial, analysis of data, interpretation of results or the preparation of this manuscript.

\section{Financial Disclosure Statement:}

Dr S Ladhani and Prof P T Heath have conducted studies on behalf of St George's, University of London funded by vaccine manufacturers but do not receive any personal payments or travel support.

Prof A J Pollard has previously conducted clinical trials on behalf of Oxford University, funded by vaccine manufacturers but did not receive any personal payments from them. Prof A J Pollard chairs the UK Department of Health's (DH) Joint Committee on Vaccination and Immunization (JCVI); the views expressed in this manuscript do not necessarily reflect the views of JCVI or DH.

Dr S N Faust acts as chief or principal investigators for clinical trials and studies conducted on behalf of University Hospital Southampton NHS Foundation Trust and the University of Southampton, sponsored by vaccine manufacturers, Universities or NHS Trusts, but receives no personal payments from them. Dr SN Faust has participated in advisory boards for vaccine manufacturers, but receives no personal payments for this work. All grants and honoraria are paid into accounts at the NHS Trust or University.

Prof D Goldblatt: UCL ICH receives funding for contract research from GSK. Prof D Goldblatt contributes to occasional GSK advisory boards. D Goldblatt is supported by the National Institute for Health Research Biomedical Research Centre at Great Ormond Street Hospital for Children NHS Foundation Trust and University College London.

All other authors have no financial relationships relevant to this article to disclose.

\section{Conflict of Interest Statement:}

Dr S Ladhani and Prof P T Heath have conducted studies on behalf of St George's, University of London funded by vaccine manufacturers but do not receive any personal payments or travel support.

Prof A J Pollard has previously conducted clinical trials on behalf of Oxford University, funded by vaccine manufacturers but did not receive any personal payments from them. Prof A J Pollard chairs the UK Department of Health's (DH) Joint Committee on Vaccination and Immunization (JCVI); the views expressed in this manuscript do not necessarily reflect the views of JCVI or DH.

Dr S N Faust acts as chief or principal investigators for clinical trials and studies conducted on behalf of University Hospital Southampton NHS Foundation Trust and the University of Southampton, sponsored by vaccine manufacturers, Universities or NHS Trusts, but receives 
no personal payments from them. Dr SN Faust has participated in advisory boards for vaccine manufacturers.

Prof D Goldblatt: Prof D Goldblatt contributes to occasional GSK advisory boards. D Goldblatt is supported by the National Institute for Health Research Biomedical Research Centre at Great Ormond Street Hospital for Children NHS Foundation Trust and University College London.

All other authors have no conflicts of interest relevant to this article to disclose

$\begin{array}{ll}\text { Abbreviations: } & \\ \text { PCV7 } & 7 \text { valent pneumococcal conjugate vaccine } \\ \text { PCV13 } & 13 \text { valent pneumococcal conjugate vaccine } \\ \text { IgG } & \text { Immunoglobulin G } \\ \text { GMC } & \text { Geometric mean concentrations } \\ \text { IPD } & \text { Invasive pneumococcal disease }\end{array}$

What's known on this subject:

Premature infants have a higher risk of invasive pneumococcal disease and are more likely to have lower vaccine responses compared to term infants. The optimal primary schedule to generate protective concentrations of pneumococcal antibodies in preterm infants is unknown.

What this study adds:

This 13-valent pneumococcal conjugate vaccine schedule RCT in preterm infants demonstrated that a reduced primary schedule resulted in higher post-booster, but lower postprimary IgG concentrations. The optimum schedule for preterm infants depends on when they are most at risk of invasive disease. 


\section{Contributors' Statement Page:}

Dr A Kent coordinated the study, performed statistical analysis and drafted the initial manuscript.

Dr S Ladhani assisted with the design of the study, coordination of the study, critically reviewed the manuscript and approved the final manuscript as submitted.

Dr N Andrews approved the data collection tools, performed the statistically analysis, critically reviewed the manuscript and approved the final manuscript as submitted.

Dr T Scorrer, Prof A Pollard, Dr P Clarke, Dr S Hughes, Dr C Heal, Dr E Menson, Dr J Chang, Dr P Satodia, Dr A C Collinson and Dr S Faust were members of the trial steering committee, recruited participants and were responsible for data collection and study procedures at their sites. They critically reviewed the manuscript and approved the final manuscript as submitted.

Prof D Goldblatt supervised the analysis of all laboratory samples, critically reviewed the manuscript and approved the final manuscript as submitted.

Prof E Miller and Prof P T Heath were responsible for the concept and design of the study and the overall supervision of all aspects of the clinical trial. They critically reviewed the manuscript and approved the final manuscript as submitted. 


\section{Abstract}

\section{Background}

Premature infants have a higher risk of invasive pneumococcal disease and are more likely to have lower vaccine responses compared to term infants. Increasingly, immunization schedules are including a reduced, 2-dose, pneumococcal conjugate vaccine (PCV) priming schedule.

We aimed to assess the immunogenicity of 3 commonly used PCV13 priming schedules in premature infants, and their response to a 12-month booster dose.

\section{Methods}

Premature infants ( $<35$ weeks gestation) were randomized to receive PCV13 at 2 and 4 months (reduced schedule); 2, 3 and 4 months (accelerated schedule); or 2, 4 and 6 months (extended schedule). All infants received a 12-month PCV13 booster. Serotype-specific pneumococcal immunoglobulin G (IgG) for PCV13 serotypes were measured by ELISA 1 month after primary and booster vaccinations.

\section{Results}

A total of 210 infants (median birth gestation $29^{+6}$ weeks, range $23^{+2}-34^{+6}$ ) were included. Following primary vaccination, $75 \%$ (95\% CI 62-85), 88\% (95\% CI 76-95) and 97\% (95\% CI 87-99) of participants had protective antibody concentrations for at least half the PCV13 serotypes for the reduced, accelerated and extended schedules respectively. Following booster vaccination, participants receiving the extended schedule had significantly lower $(\mathrm{p}<0.05)$ geometric mean concentrations compared with reduced (for 9/13 serotypes) and accelerated schedules (for 4/13 serotypes), but nearly all participations, regardless of schedule or serotype, had seroprotective IgG concentrations.

\section{Conclusions}

A reduced priming schedule of PCV13 resulted in higher post-booster IgG concentrations, but lower post-primary concentrations. The optimum vaccine schedule for preterm infants will therefore depend on when they are most at risk of invasive pneumococcal disease. 


\section{Introduction}

Premature infants are at increased risk of vaccine preventable diseases, including a two-fold risk of invasive pneumococcal disease (IPD) compared to term infants.[1-3]

In most industrialised countries with established pneumococcal immunization programmes, the 13-valent pneumococcal conjugate vaccine (PCV13) has superseded the 7-valent PCV and has been shown to be highly immunogenic in term infants.[4-6]

The immunogenicity of PCV13 in premature infants receiving a 2-3-4 and 12-month schedule was only recently reported and showed lower immunoglobulin $\mathrm{G}(\mathrm{IgG})$ concentrations for 8 serotypes after both primary and booster doses compared to term infants. [7] This lower immunogenicity is consistent with previous PCV7 studies [8-10] and is concerning because premature infants are also less likely to benefit from the protective maternal antibodies transferred during late pregnancy.

Additionally, national immunization programmes are increasingly including reduced (2) dose priming schedules.[11,12] These schedules are immunogenic in term infants and, with some vaccines, may even improve B cell memory and booster responses.[13-16] However, little is known about the immunogenicity of fewer primary doses in premature infants.

This randomized, controlled trial aimed to assess the immunogenicity of reduced, accelerated (intended to provide maximum early protection) and extended (doses administered over a longer period) PCV13 priming schedules in premature infants after completion of the primary series and after a 12-month booster. 


\section{Patients and Methods}

\section{Participants and recruitment}

Premature infants were enrolled in a phase IV open-label randomized controlled trial from 12 UK centres between May 2012 and May 2013. Potentially eligible infants were identified by the clinical teams and parents were provided with information by the research teams. Infants were eligible for inclusion if they had a birth gestation less than $35^{+0}$ weeks, had no contraindications for vaccination as defined by Department of Health guidelines[17] and were between 7 and 12 weeks of age. Additionally, infants should not have received any other vaccinations (with the exceptions of BCG and hepatitis B). Information on the participants' past medical, medication and vaccination history was collected from the medical records using a standardised case report form.

Written informed consent was obtained from parents prior to enrolment. The study was approved by the East of England - Essex research ethics committee (REC reference 07/HO301.11) and registered on the EudraCT clinical trial database (2007-007535-23).

\section{Vaccination}

Infants were randomly assigned (1:1:1) to receive PCV13 (Prevenar13; Pfizer, New York) at 2 and 4 months of age (reduced schedule - Group 1), at 2, 3 and 4 months of age (accelerated schedule - Group 2) or at 2, 4 and 6 months of age (extended schedule - Group 3)(supplementary table 1). A booster dose of PCV13 was administered to all infants at 12 months of age. Additionally, all participants received a combined diphtheria, tetanus, acellular pertussis, Haemophilus influenzae type b and inactivated polio vaccine (Pediacel; Sanofi Pasteur MSD, Lyon, France) at 2, 3 and 4 months old, meningococcal C-CRM 197 vaccine (Menjugate; Novartis Vaccines, Siena, Italy) at 3 and 4 months of age and a combined measles, mumps and rubella vaccine (Priorix; GlaxoSmithKline Biologicals, 
Rixensart, Belgium) and Hib-MenC-TT conjugate vaccine (Menitorix, GlaxoSmithKline Biologicals, Rixensart, Belgium) at 12 months of age (supplementary table 1). Participants were vaccinated in hospital if still receiving inpatient care. All vaccines were administered intramuscularly.

Computerised block randomization was stratified by centre and gestation $(<30$ or $\geq 30$ weeks gestation) and each centre was allocated blocks of sequential numbers (block size 18). Following consent the subject was allocated the next available study number for that centre and gestational age cohort, and the appropriate sealed envelope containing the group allocation opened. The study was not blinded to parents or clinical personnel.

\section{Blood sampling and serological methods}

Up to $3 \mathrm{~mL}$ of whole blood was obtained from each participant prior to the first vaccination (baseline), 1 month following primary vaccination (at age 5 months for Groups 1 and 2 participants and at age 7 months for Group 3 participants), prior to and 1 month after booster vaccination (12 and 13 months respectively) (supplementary table 1).

Serological analysis was performed at the World Health Organisation reference laboratory for pneumococcal serology, Institute of Child Health, London. Following extraction from whole blood, sera were stored at $-70^{\circ} \mathrm{C}$ prior to assay for pneumococcal serotype-specific immunoglobulin $\mathrm{G}$ (IgG) concentrations for the PCV13 pneumococcal serotypes by enzymelinked immunosorbent assay (ELISA) as previously described.[18] The lower limit of assay quantification was $0.15 \mu \mathrm{g} / \mathrm{mL}$ and IgG concentrations $\geq 0.35 \mu \mathrm{g} / \mathrm{ml}$ were considered protective.[19]

\section{Safety analysis}


All participants were observed for immediate adverse reactions. Solicited systemic and local adverse reactions were recorded by the infant's main caregiver for 7 days following each vaccination. All AEs (including serious adverse events) were recorded for 28 days after each vaccination using an adverse event (AE) diary. Parents had access to a 24-hour telephone contact number for AE reporting.

\section{Statistical analysis}

The primary objectives were to assess IgG geometric mean concentrations (GMCs) and the proportion of infants with protective serotype-specific antibody concentrations for PCV13 serotypes at 1 month after completion of the primary vaccination course, according to the 3 schedules. The main secondary objectives were to assess differences in serotype-specific IgG GMC and seroprotection rates between schedules prior to and following booster vaccination at 12 months of age; and to quantify the percentage of children experiencing fever, local reactions and non-febrile systemic reactions within 7 days following each vaccine dose. Pre-trial sample size calculations estimated a minimum of 60 infants in each group to detect at least a 2 fold difference between groups after primary immunization, with $80 \%$ power and 5\% significance. Based on published data, the standard deviation of $\operatorname{IgG}$ responses was estimated be $0.6 \log _{10}$ units.[20] To allow for drop out of subjects over the course of the study and the challenges of obtaining blood samples from very premature infants, we aimed to recruit 210 infants.

Data were analyzed using a modified intention to treat analysis including all infants who received a dose of PCV13 and from whom at least one post-vaccination blood sample was obtained. GMCs and 95\% confidence intervals (CI) were calculated for each sampling time point, along with the proportion of infants achieving protective antibody concentrations and 
binominal CI. Results below the lower limit of quantification (LLQ) were taken to be half the LLQ for computational purposes.

Statistical comparison of antibody concentrations and the proportion of participants with protective concentrations or AEs between the 3 trial arms were performed using the Student's t-test and the $\chi 2$-test or Fisher's exact test, as appropriate. Statistical significance was defined as $\mathrm{p}<0.05$. To facilitate comparisons we have analysed schedules based on the proportions achieving adequate protection for at least half of the serotypes. The number of serotypes with protective concentrations per participant were compared using the non-parametric KruskalWallis one-way analysis of variance test.

Logistic regression was used to examine the effect of gestation, the receipt of antenatal or postnatal steroids, blood transfusion, BCG vaccination, early post-vaccination paracetamol and the presence of chronic lung disease (CLD, defined as requiring oxygen or respiratory support at 28 days of age) on seroprotection. Analysis was adjusted for gestation. For postprimary vaccination results multivariable linear regression using log-transformed values was performed (adjusting for group and gestation). Linear regression was not performed on baseline IgG concentrations due to the large number of results below the LLQ.

All data were analyzed using STATA version 13 (Stata Inc). 


\section{Results}

A total of 210 infants were recruited. 199 participants (94.7\%) completed the primary phase (primary endpoint) and 194 (92.4\%) completed the entire study (Figure 1). 2 participants died of causes unrelated to the trial. The majority of infants who did not meet the inclusion criteria were outside the study age range or were too unstable for vaccination. A second group of infants was excluded for logistical reasons - many were transferred to their local neonatal unit prior to their first vaccination (Figure 1).

The characteristics of randomized infants were similar between groups (Table 1) with a median birth gestation of $29^{+6}$ (range, $23^{+2}-34^{+6}$ ) weeks and median birth weight of $1388 \mathrm{~g}$ (range: $450-3390 \mathrm{~g}$ ). 112 vaccinations were administered to hospitalized participants.

\section{Primary vaccination}

At baseline participants had very low antibody concentrations for all pneumococcal serotypes (Table 2, supplementary table 2). The highest IgG GMCs (for all participants) were seen for serotypes $14(0.26 \mu \mathrm{g} / \mathrm{mL})$ and 19A $(0.19 \mu \mathrm{g} / \mathrm{mL})$.

Following the primary vaccination course, substantial increases in antibody concentrations were seen for all serotypes and all groups. There was considerable variation between serotypes with IgG GMCs ranging from $0.16 \mu \mathrm{g} / \mathrm{mL}$ for serotype $6 \mathrm{~B}$ (reduced schedule) to $8.49 \mu \mathrm{g} / \mathrm{mL}$ for serotype 14 (extended schedule) (Figure 2; Supplementary Table 3).

The primary schedule had a significant impact on vaccine immunogenicity. Lack of seroprotection for more than half the PCV13 serotypes was seen in $25 \%, 12 \%$ and $3 \%$ of participants receiving the reduced, accelerated and extended schedules respectively $(\mathrm{p}<0.001$, supplementary figure 1 and supplementary table 4). 
Participants receiving the extended schedule had higher IgG GMCs compared with the reduced schedule for 11 serotypes and accelerated schedule for 7 serotypes. The accelerated schedule was superior to the reduced schedule for 4 serotypes (Figure 2, Table 2; Supplementary table 3).

\section{Booster vaccination}

At 12 months of age, waning of pneumococcal antibody concentrations was evident with low rates of seroprotection against individual serotypes (Table 3; Supplementary table 5). Antibody concentrations remained significantly higher in those who had received the extended schedule compared with reduced (for 10 serotypes) or accelerated schedules (for 11 serotypes), the accelerated schedule was superior to the reduced schedule for one serotype only.

Following booster vaccination a high proportion of infants achieved protective concentrations (Table 3). As at previous time points, significant variation in antibody concentrations between serotypes and groups was apparent (Figure 3). In contrast to post-primary vaccination responses, participants receiving the extended schedule had lower GMCs compared with the reduced (for 9 serotypes) and accelerated schedules (for 4 serotypes). The accelerated schedule was inferior to the reduced schedule for one serotype (19A) (supplementary table 6). Infants who received the extended schedule had lower fold increases in concentrations following booster vaccination than the other groups (supplementary figure 2).

\section{Predictors of antibody concentrations}


Increased odds of seroprotection at 2 months of age were seen with each week of increased gestation for 4 serotypes: 6A (OR 1.34, 95\% CI 1.12-1.60; $\mathrm{p}=0.001), 14$ (OR 1.25, 95\% CI $1.12-1.41 ; \mathrm{p}<0.001), 19 \mathrm{~A}$ (OR 1.27, 95\% CI 1.12-1.45; $\mathrm{p}<0.001)$ and 19F (OR 1.29, 95\% CI 1.09-1.52; $\mathrm{p}=0.003)$. Later gestation was associated with an increase in post primary vaccination $\operatorname{IgG}$ concentrations for 3 serotypes: 1 (6\% increase per week, 95\% CI 0.9-12; $\mathrm{p}=0.021), 3(8 \%$ increase per week, $95 \%$ CI 4-14, $\mathrm{p}<0.001)$ and $7 \mathrm{~F}(8 \%$ increase per week, 95\% CI 3-13; $\mathrm{p}=0.002)$.

Receipt of antenatal steroids was associated with decreased odds of seroprotection at 2 months for 4 serotypes: 5 (OR 0.09, 95\% CI 0.01-0.83; $\mathrm{p}=0.033), 6 \mathrm{~A}(\mathrm{OR} 0.26,95 \%$ CI 0.10 $0.69 ; \mathrm{p}=0.006), 19 \mathrm{~A}(\mathrm{OR} 0.19,95 \%$ CI $0.08-0.45 ; \mathrm{p}<0.001$ and $23 \mathrm{~F}$ (OR 0.23, 95\% CI 0.060.80, $\mathrm{p}=0.021$ ). Additionally, post-primary vaccination serotype-specific IgG GMCs for serotypes 1,4 and $9 \mathrm{~V}$ were reduced in infants who had been exposed to antenatal steroids. At no time-points were antenatal steroids associated with higher antibody concentrations.

Pre- or post-primary protective concentrations were not associated with any other factors in regression analysis. An insufficient number of infants (14) received postnatal steroids to analyse any effect. Serotype-specific antibody concentrations after the 12-month PCV13 booster were affected by priming schedule and pre-existing antibody levels only.

\section{Safety and adverse events}

There were no significant differences in the frequency or severity of local and systemic AEs between vaccination schedules at any time-point. Altogether 77 serious adverse events (SAEs) were reported (including the 2 deaths). SAEs were predominantly acute respiratory infections. There was 1 possibly related (suspected) unexpected serious adverse reaction 
from each randomized group: 2 participants had necrotising enterocolitis within a week of vaccination and 1 participant had post-vaccination cardiorespiratory instability requiring readmission; all 3 infants made a good recovery.

\section{Discussion}

This is the first study to compare different PCV13 schedules in premature infants and demonstrates the need for early and effective immunization strategies for this vulnerable group, given their very low pre-immunization antibody concentrations. Our results indicate that most preterm infants can achieve seroprotective antibody concentrations for the serotypes in PCV13 regardless of the primary schedule administered, especially after the 12month booster, but the magnitude of their immunological response is dependent on the primary schedule they receive.

Serotype-specific responses varied, with lower IgG GMCs achieved for serotypes 3, 5 and 6B after the primary course and for serotypes $3,9 \mathrm{~V}$ and $18 \mathrm{C}$ after the booster dose; these findings are consistent with those observed in term infants.[4,21] However, when compared with previous term (PCV13) and preterm (PCV7) studies, antibody concentrations after primary and booster vaccination are lower overall, resulting in lower seroprotection following primary vaccination. $[4,5,8,9,22]$

Similarly, compared with the recent PCV13 preterm study[7], lower IgG GMCs and seroprotection rates were seen for all serotypes. These differences may be due to the different laboratory testing methodology for serotype-specific antibody concentrations, but potential biological explanations include interactions with concurrently administered vaccines, the younger gestation of our cohort or our broad inclusion criteria encompassing 
infants with complex medical problems - representative of the preterm population.

Additionally, Martinon-Torres et al. did not report baseline IgG concentrations which may differ between countries and impact on post-vaccination concentrations.[7]

When comparing schedules within our cohort, the most striking finding was the contrasting immunogenicity of the 3 schedules at different time points, with the reduced dose schedule generating inferior antibody concentrations after the primary course but superior antibody concentrations after the booster dose. The higher post-primary IgG GMCs following 3 doses (compared with 2 doses) is consistent with two meta-analyses of primary schedules in term infants.[23,24] Of the 3-dose schedules, higher antibody concentrations were seen in premature infants receiving the extended schedule. This was not observed in the metaanalyses of term infant responses but an older age at final vaccination may be more important in premature infants as it will allow further maturation of their immune system.[25,26] However, this needs to be set against the optimal age at which protection is required in this population. Several studies have indicated an increased susceptibility of IPD in babies born prematurely when compared with term infants; this risk appears maximal in the first 6 months of life.[1-3]

The differences in response to the booster dose was unexpected as the type of priming schedule has not been consistently shown to affect the generation of immunological memory and PCV booster vaccine responses in term infants.[23,27] The improved post-booster immunogenicity of fewer priming doses is well described for meningococcal $\mathrm{C}$ conjugate vaccines and is thought to be due to lower total antigen exposure favouring differentiation of B lymphoblasts into memory B cells instead of antibody-generating plasma cells.[14,15] In pneumococcal conjugate vaccines, a study of Fijian infants receiving one PCV7 priming dose 
followed by the 23-valent pneumococcal polysaccharide vaccine (PPV23) at 12 months had higher IgG GMC for serotypes 4, 9V, 19F compared with those who had been primed with two or three PCV7 doses.[13] Similarly, infants receiving a lower antigen-containing investigational tetravalent PCV for priming had higher booster responses than those who had received the higher antigen-containing preparation.[28] However, it should be noted that a statistically significant difference between the reduced and accelerated schedule groups was observed for only one serotype.

Despite seroprotective concentrations, infants who had received the extended schedule had lower fold increases in antibody concentrations following booster vaccination than those receiving either the reduced dose or accelerated schedules suggesting that the higher prebooster antibody concentrations at 12 months may have interfered with booster responses. This effect has been observed following booster doses for other vaccines and several hypotheses have been proposed including the formation of immune complexes consisting of pre-existing antibody and vaccine antigen resulting in less available vaccine antigen, and $\mathrm{B}$ cell receptor mediated negative feedback mechanisms, analogous to those described for high maternal antibody concentrations impairing primary vaccine responses.[29-33]

Within our cohort of premature infants, increasing birth gestation was associated with increased immunogenicity. This has previously been described for other vaccines and reflects deficiencies in both the innate and adaptive immune systems in these more premature infants.[34-39]

\section{Limitations}

The study had some potential limitations. The different ages of infants at blood sampling between the groups must be considered when comparing primary schedules; the antibody 
concentrations at 7 months for babies in Groups 1 and 2 are not known. It is possible, that infants in those groups may have had a rise in their antibody concentrations between their 5 month sample and 7 months of age due to natural exposure.[40] However, a recent study comparing schedules in term infants which sampled some infants at both 5 and 8 months did not find a rise in antibodies between these ages.[27] We also did not measure antibody concentrations beyond 13 months of age.

As the objectives of this study were to look at schedule differences within the premature population we did not include a term comparator group, however lower antibody concentrations were seen in our cohort when compared with a recent cohort of term infants in the UK who received a reduced dose schedule, which was analyzed in the same laboratory.[22]

Additionally, we did not include any assessment of functional activity of the antibodies detected. Opsonophagocytic antibody titres may have allowed us to assess the potential clinical impact of schedule differences in more detail and should be considered in future studies. A previous meta-analysis of primary PCV schedules in term infants has shown a good relationship between ELISA measured IgG concentrations and opsonophagocytic antibody titres, however an analysis of serotype-specific OPA values did not find a consistent protective OPA titre across all vaccine serotypes.[24,41]

\section{Conclusion}

PCV13 is well tolerated in premature infants. Different priming schedules result in higher IgG concentrations at different times during the first 13 months of life. We believe that such data will be of benefit to those planning or providing pneumococcal vaccines to preterm infants and will enable them to consider this in the context of their own immunization programmes and epidemiological situations. 



\section{Funding}

This work was supported by Pfizer Ltd as an investigator-led study. The funder had no input into the conduct of the trial, analysis of data, interpretation of results or the preparation of this manuscript. 


\section{Acknowledgements section}

\section{Acknowledgements}

We would like to thank all children who took part in the study as well as their parents/guardians, Dr Nicola Pritchard and the study staff at all the research centres, Pauline Kaye, Deborah Cohen, Teresa Gibbs and all other members of the team at Public Health England and NIHR CRN for their invaluable help and support and Pfizer for financially supporting this investigator-led study.

\section{PUNS study group}

Ager, Gill (1)

Snape, Matthew D (2)

Few, Karen (3)

Varghese, Anu S (4)

Reynolds, Sarah (5)

Bromage, Barbara (6)

Blake, Elizabeth (7)

Burbridge, Polly (8)

Thalasselis, Vasili (8)

England, Anna (9)

Mary Matheson (9)

Pauline Waight (10)

1. Neonatal Unit, Queen Alexandra Hospital, Portsmouth, UK

2. Oxford Vaccine Group, University of Oxford, and the NIHR Oxford Biomedical Research Centre, Oxford UK

3. Neonatal Unit, Norfolk and Norwich University Hospitals NHS Foundation Trust, Norwich, UK

4. Department of Immunology, Royal Manchester Children's Hospital, Manchester, UK

5. Neonatal Unit, University Hospital Coventry and Warwickshire NHS Trust, Coventry, UK

6. Neonatal Unit, Royal Cornwall Hospital, Truro, UK

7. University of Southampton and University Hospital Southampton NHS Foundation Trust

8. Institute of Child Health, UCL, London, UK

9. Immunoassay Laboratory, Public Health England, Porton Down, UK

10. Immunization, Hepatitis and Blood Safety Department, Public Health England, Colindale, London, UK

\section{Previously presented in part:}

Abstract 460: May 2014, ESPID annual meeting, Dublin, Ireland Abstract 240: May 2015, ESPID annual meeting, Leipzig, Germany 


\section{REFERENCES}

1. Rückinger S, van der Linden M, von Kries R. Effect of heptavalent pneumococcal conjugate vaccination on invasive pneumococcal disease in preterm born infants. BMC Infect. Dis. 2010; 10:12.

2. Hjuler T, Wohlfahrt J, Simonsen J, et al. Perinatal and crowding-related risk factors for invasive pneumococcal disease in infants and young children: a population-based casecontrol study. Clin. Infect. Dis. 2007; 44:1051-6.

3. Shinefield H, Black S, Ray P, Fireman B, Schwalbe J, Lewis E. Efficacy, immunogenicity and safety of heptavalent pneumococcal conjugate vaccine in low birth weight and preterm infants. Pediatr. Infect. Dis. J. 2002; 21:182-6.

4. Snape MD, Klinger CL, Daniels ED, et al. Immunogenicity and Reactogenicity of a 13-Valent-pneumococcal Conjugate Vaccine Administered at 2, 4, and 12 Months of Age. Pediatr. Infect. Dis. J. 2010; 29:e80-e90.

5. Grant LR, O'Brien SE, Burbidge $\mathrm{P}$, et al. Comparative immunogenicity of 7 and 13 valent pneumococcal conjugate vaccines and the development of functional antibodies to cross-reactive serotypes. PLoS One 2013; 8:e74906.

6. Miller E, Andrews NJ, Waight PA, Slack MPE, George RC. Effectiveness of the new serotypes in the 13-valent pneumococcal conjugate vaccine. Vaccine 2011; 29:912731.

7. Martinón-Torres F, Czajka H, Wysocki J. 13-Valent Pneumococcal Conjugate Vaccine ( PCV13 ) in Preterm Versus Term Infants. Pediatrics 2015; 135.

8. Ruggeberg JU, Collins C, Clarke P, et al. Immunogenicity and induction of immunological memory of the heptavalent pneumococcal conjugate vaccine in preterm UK infants. Vaccine 2007; 25:264-71.

9. Moss SJ, Fenton AC, Toomey JA, Grainger AJ, Smith J, Gennery AR. Responses to a conjugate pneumococcal vaccine in preterm infants immunized at 2, 3, and 4 months of age. Clin. Vaccine Immunol. 2010; 17:1810-6.

10. Bonhoeffer J, Siegrist C, Heath PT. Immunisation of premature infants. Arch. Dis. Child. 2006; 91:929-35.

11. Flasche S, Hoek AJ Van, Goldblatt D, et al. The Potential for Reducing the Number of Pneumococcal Conjugate Vaccine Doses While Sustaining Herd Immunity in HighIncome Countries. PloS Med. 2015; 12:e1001839.

12. Findlow $\mathrm{H}$, Borrow $\mathrm{R}$. Is a single infant priming dose of meningococcal serogroup $\mathrm{C}$ conjugate vaccine in the United Kingdom sufficient? Hum. Vaccin. Immunother. 2015; 11:1501-6.

13. Russell FM, Licciardi P V, Balloch A, et al. Safety and immunogenicity of the 23valent pneumococcal polysaccharide vaccine at 12 months of age, following one, two, 
or three doses of the 7-valent pneumococcal conjugate vaccine in infancy. Vaccine 2010; 28:3086-94.

14. Richmond P, Borrow R, Miller E, et al. Meningococcal Serogroup C Conjugate Vaccine Is Immunogenic in Infancy and Primes for Memory. J. Infect. Dis. 1999; 179:1569-1572.

15. Borrow R, Goldblatt D, Finn A, et al. Immunogenicity of, and Immunologic Memory to, a Reduced Primary Schedule of Meningococcal C-Tetanus Toxoid Conjugate Vaccine in Infants in the United Kingdom. Infect. Immun. 2003; 71:5549-5555.

16. Pace D, Khatami A, McKenna J, et al. Immunogenicity of reduced dose priming schedules of serogroup $\mathrm{C}$ meningococcal conjugate vaccine followed by a booster at 12 months in infants: an open label randomised controlled trial. Br. Med. J. (Clin. Res. Ed). 2015; 350:h1554.

17. Department of Health. Contraindications and special considerations (Chapter 6). Immunisation against Infectious Disease: The Green Book. London, UK: The Stationary Office, 2006. Available at: https://www.gov.uk/government/collections/immunisation-against-infectious-diseasethe-green-book.

18. Concepcion NF, Frasch CE. Pneumococcal Type 22F Polysaccharide Absorption Improves the Specificity of a Enzyme-Linked Immunosorbent Assay. Clin. Vaccine Immunol. 2001; 8:266-272.

19. Jódar L, Butler J, Carlone G, et al. Serological criteria for evaluation and licensure of new pneumococcal conjugate vaccine formulations for use in infants. Vaccine 2003; $21: 3265-3272$.

20. Goldblatt D, Southern J, Ashton L, et al. Immunogenicity of a reduced schedule of pneumococcal conjugate vaccine in healthy infants and correlates of protection for serotype 6B in the United Kingdom. Pediatr. Infect. Dis. J. 2010; 29:401-5.

21. Goldblatt D, Southern J, Ashton L, et al. Immunogenicity and boosting after a reduced number of doses of a pneumococcal conjugate vaccine in infants and toddlers. Pediatr. Infect. Dis. J. 2006; 25:312-9.

22. Ladhani SN, Andrews NJ, Waight $\mathrm{P}$, et al. Interchangeability of meningococcal group $\mathrm{C}$ conjugate vaccines with different carrier proteins in the United Kingdom infant immunisation schedule. Vaccine 2015; 33:648-55.

23. Knoll MD, Park DE, Johnson TS, et al. Systematic review of the effect of pneumococcal conjugate vaccine dosing schedules on immunogenicity. Pediatr. Infect. Dis. J. 2014; 33 Suppl 2:S119-29.

24. Scott P, Rutjes AWS, Bermetz L, et al. Comparing pneumococcal conjugate vaccine schedules based on 3 and 2 primary doses: systematic review and meta-analysis. Vaccine 2011; 29:9711-21. 
25. Prabhudas M, Adkins B, Gans H, et al. Challenges in infant immunity : implications for responses to infection and vaccines. Nat. Immunol. 2011; 12:189-194.

26. Siegrist C-A, Aspinall R. B-cell responses to vaccination at the extremes of age. Nat. Rev. Immunol. 2009; 9:185-94.

27. Spijkerman J, Veenhoven RH, Wijmenga-Monsuur AJ, et al. Immunogenicity of 13valent pneumococcal conjugate vaccine administered according to 4 different primary immunization schedules in infants: a randomized clinical trial. JAMA 2013; 310:9307.

28. Åhman H, Käyhty H, Vuorela A, Leroy O, Eskola J. Dose dependency of antibody response in infants and children to pneumococcal polysaccharides conjugated to tetanus toxoid. Vaccine 1999; 17:2726-2732.

29. Danilova E, Shiryayev A, Kristoffersen EK, Sjursen H. Attenuated immune response to tetanus toxoid in young healthy men protected against tetanus. Vaccine 2005; 23:4980-3.

30. Danilova E, Shiryayev A, Skogen V, Kristoffersen EK, Sjursen H. Short-term booster effect of diphtheria toxoid in initially long-term protected individuals. Vaccine 2005; 23:1446-50.

31. Rohner GB, Snape MD, Kelly DF, et al. The Magnitude of the Antibody and Memory B Cell Responses during Priming with a Protein-Polysaccharide Conjugate Vaccine in Human Infants Is Associated with the Persistence of Antibody and the Intensity of Booster Response. J. Immunol. 2008; 180:2165-2173.

32. Andrews NJ, Walker WT, Finn A, et al. Predictors of immune response and reactogenicity to AS03B-adjuvanted split virion and non-adjuvanted whole virion H1N1 (2009) pandemic influenza vaccines. Vaccine 2011; 29:7913-9.

33. Knuf M, Schmitt H-J, Wolter J, et al. Neonatal vaccination with an acellular pertussis vaccine accelerates the acquisition of pertussis antibodies in infants. J. Pediatr. 2008; 152:655-60, 660.e1.

34. Sharma AA, Jen R, Butler A, Lavoie PM. The developing human preterm neonatal immune system: a case for more research in this area. Clin. Immunol. 2012; 145:61-8.

35. Lavoie PM, Huang Q, Jolette E, et al. Profound lack of interleukin (IL)-12/IL-23p40 in neonates born early in gestation is associated with an increased risk of sepsis. J. Infect. Dis. 2010; 202:1754-63.

36. Zhao Y, Dai Z-P, Lv P, Gao X-M. Phenotypic and functional analysis of human T lymphocytes in early second- and third-trimester fetuses. Clin. Exp. Immunol. 2002; 129:302-8.

37. Berrington JE, Barge D, Fenton AC, Cant AJ, Spickett GP. Lymphocyte subsets in term and significantly preterm UK infants in the first year of life analysed by single platform flow cytometry. Clin. Exp. Immunol. 2005; 140:289-92. 
38. McGreal EP, Hearne K, Spiller OB. Off to a slow start: under-development of the complement system in term newborns is more substantial following premature birth. Immunobiology 2012; 217:176-86.

39. Slack MH, Schapira D, Thwaites RJ, et al. Acellular pertussis vaccine given by accelerated schedule: response of preterm infants. Arch. Dis. Child. Fetal Neonatal Ed. 2004; 89:F57-60.

40. Van Hoek AJ, Sheppard CL, Andrews NJ, et al. Pneumococcal carriage in children and adults two years after introduction of the thirteen valent pneumococcal conjugate vaccine in England. Vaccine 2014; 32:4349-55.

41. Andrews NJ, Waight PA, Burbidge P, et al. Serotype-specific effectiveness and correlates of protection for the 13-valent pneumococcal conjugate vaccine: a postlicensure indirect cohort study. Lancet Infect. Dis. 2014; 3099:1-8. 
Table 1: Participant characteristics by group.

Median (range) or n (\%). CLD: Chronic lung disease. BCG: Bacillus Calmette-Guérin vaccination.

Table 1

\begin{tabular}{|l|l|l|l|}
\hline & \multicolumn{1}{|c|}{$\begin{array}{c}\text { Reduced dose } \\
\text { (Group 1) } \\
\mathrm{n}=68\end{array}$} & \multicolumn{1}{|c|}{$\begin{array}{c}\text { Accelerated } \\
\text { (Group 2) } \\
\mathrm{n}=67\end{array}$} & \multicolumn{1}{|c|}{$\begin{array}{c}\text { Extended } \\
\text { (Group 3) } \\
\mathrm{n}=71\end{array}$} \\
\hline Gestation (weeks) & $29.6(24.9-34.9)$ & $30(23.6-34.9)$ & $30(23.3-34.9)$ \\
\hline Birth weight (g) & $1410(576-2600)$ & $1360(510-3390)$ & $1390(450-2680)$ \\
\hline Weight at 1 ${ }^{\text {st }}$ vaccination (g) & $2442(845-4660)$ & $2350(1260-5070)$ & $2497(920-4560)$ \\
\hline Sex (male) & $37(54)$ & $32(48)$ & $38(54)$ \\
\hline Ethnicity (white) & $57(84)$ & $54(81)$ & $60(85)$ \\
\hline CLD & $23(34)$ & $22(33)$ & $27(38)$ \\
\hline Antenatal steroids & $59(87)$ & $56(84)$ & $62(87)$ \\
\hline Postnatal steroids & $4(6)$ & $4(6)$ & $6(8)$ \\
\hline Blood transfusion & $28(41)$ & $30(45)$ & $29(41)$ \\
\hline BCG & $5(7)$ & $5(7)$ & $7(10)$ \\
\hline Age at visit 1 (days) & $61(49-86)$ & $61(49-83)$ & $61(46-88)$ \\
\hline Age at visit 2 (days) & $93(78-136)$ & $93(82-119)$ & $95(79-132)$ \\
\hline Age at visit 3 (days) & $126(111-178)$ & $126(114-160)$ & $126(106-160)$ \\
\hline Age at visit 4 (days) & $158(132-199)$ & $158(135-187)$ & - \\
\hline Age at visit 5 (days) & - & - & $181(156-258)$ \\
\hline Age at visit 6 (days) & - & - & $209(177-298)$ \\
\hline Age at visit 7 (days) & $368(353-410)$ & $367(351-404)$ & $368(351-429)$ \\
\hline Age at visit 8 (days) & $400(367-443)$ & $400(376-492)$ & $397(375-606)$ \\
\hline
\end{tabular}


Table 2: Proportion of infants with protective antibody concentrations ( $\operatorname{IgG} \geq 0.35 \mu \mathrm{g} / \mathrm{mL}$ ) for the 13 PCV13 serotypes at baseline and 1 month after final primary vaccination.

Proportion $(95 \% \mathrm{CI})$. a b c: $\mathrm{p}<0.05$ comparing reduced and accelerated, accelerated and extended, and reduced and extended schedules respectively; ${ }^{*} \mathrm{p}<0.001$

Table 2

\begin{tabular}{|l|l|l|l|l|}
\hline \multirow{3}{*}{ Serotype } & Baseline & \multicolumn{3}{|c|}{ Post primary immunization } \\
\cline { 2 - 6 } & $\begin{array}{c}\text { All } \\
\mathrm{N}=197\end{array}$ & $\begin{array}{c}\text { Reduced dose } \\
\text { (Group 1) } \\
\mathrm{N}=66\end{array}$ & $\begin{array}{c}\text { Accelerated } \\
(\text { Group 2) } \\
\mathrm{N}=60\end{array}$ & $\begin{array}{c}\text { Extended } \\
\text { (Group 3) } \\
\mathrm{N}=69\end{array}$ \\
\hline 1 & $0.03(0.01-0.07)$ & $0.85(0.74-0.92)$ & $0.80(0.68-0.89)^{b}$ & $0.94(0.86-0.98)$ \\
\hline 3 & $0.01(0.00-0.03)$ & $0.61(0.48-0.73)$ & $0.66(0.53-0.78)$ & $0.80(0.68-0.88)^{c}$ \\
\hline 4 & $0.02(0.01-0.05)$ & $0.92(0.83-0.97)$ & $0.88(0.77-0.95)$ & $0.94(0.86-0.98)$ \\
\hline 5 & $0.02(0.01-0.05)$ & $0.36(0.25-0.49)$ & $0.47(0.34-0.60)^{b}$ & $0.74(0.62-0.84)^{c^{*}}$ \\
\hline $6 \mathrm{~A}$ & $0.13(0.09-0.19)$ & $0.58(0.45-0.70)$ & $0.72(0.59-0.83)^{b *}$ & $0.94(0.86-0.98)^{c^{*}}$ \\
\hline 6B & $0.07(0.04-0.11)$ & $0.20(0.11-0.31)^{a^{*}}$ & $0.52(0.38-0.65)^{b}$ & $0.78(0.66-0.87)^{c^{*}}$ \\
\hline 7F & $0.05(0.02-0.09)$ & $0.91(0.81-0.97)$ & $0.97(0.88-1.00)$ & $1.00(0.95-1.00)^{c^{*}}$ \\
\hline 9V & $0.06(0.03-0.10)$ & $0.59(0.46-0.71)^{a}$ & $0.85(0.73-0.93)$ & $0.93(0.84-0.98)^{c^{*}}$ \\
\hline 14 & $0.38(0.31-0.45)$ & $0.94(0.85-0.98)$ & $0.98(0.91-1.00)$ & $0.99(0.92-1.00)$ \\
\hline 18C & $0.05(0.02-0.08)$ & $0.88(0.78-0.95)$ & $0.87(0.75-0.94)$ & $0.96(0.88-0.99)$ \\
\hline 19A & $0.24(0.18-0.30)$ & $0.83(0.72-0.91)^{a}$ & $0.95(0.86-0.99)$ & $0.96(0.88-0.99)^{c}$ \\
\hline 19F & $0.14(0.09-0.19)$ & $0.97(0.89-1.00)$ & $1.00(0.94-1.00)$ & $1.00(0.95-1.00)$ \\
\hline 23F & $0.06(0.03-0.10)$ & $0.47(0.34-0.60)$ & $0.63(0.50-0.75)^{b}$ & $0.83(0.72-0.91)^{c^{*}}$ \\
\hline
\end{tabular}


Table 3: Proportion of infants with protective antibody concentrations $(\operatorname{IgG} \geq 0.35 \mu \mathrm{g} / \mathrm{mL})$ prior to booster vaccination (12 months) and 1 month after booster vaccination. Proportion $(95 \% \mathrm{CI})$. a b c: $\mathrm{p}<0.05$ comparing reduced and accelerated, accelerated and extended, and reduced and extended schedules respectively; ${ }^{*} \mathrm{p}<0.001$

Table 3

\begin{tabular}{|l|l|l|l|l|c|c|}
\hline \multirow{3}{*}{ Serotype } & \multicolumn{3}{|c|}{ Pre-booster vaccination } & \multicolumn{3}{c|}{ Post booster vaccination } \\
\cline { 2 - 7 } & $\begin{array}{c}\text { Reduced dose } \\
(\text { Group 1) } \\
\mathrm{N}=64\end{array}$ & $\begin{array}{c}\text { Accelerated } \\
(\text { Group 2) } \\
\mathrm{N}=57\end{array}$ & $\begin{array}{c}\text { Extended } \\
(\text { Group 3) } \\
\mathrm{N}=69\end{array}$ & $\begin{array}{c}\text { Reduced dose } \\
\text { (Group 1) } \\
\mathrm{N}=64\end{array}$ & $\begin{array}{c}\text { Accelerated } \\
\text { (Group 2) } \\
\mathrm{N}=59\end{array}$ & $\begin{array}{c}\text { Extended } \\
\text { (Group 3) } \\
\mathrm{N}=68\end{array}$ \\
\hline 1 & $0.23(0.14-0.36)$ & $0.19(0.10-0.32)^{b^{*}}$ & $0.49(0.37-0.62)^{c}$ & $0.98(0.92-1.00)$ & $1.00(0.94-1.00)$ & $1.00(0.95-1.00)$ \\
\hline 3 & $0.18(0.09-0.30)$ & $0.22(0.12-0.35)$ & $0.29(0.18-0.41)$ & $0.89(0.78-0.95)$ & $0.93(0.83-0.98)$ & $0.87(0.76-0.94)$ \\
\hline 4 & $0.11(0.05-0.21)$ & $0.11(0.04-0.22)^{b}$ & $0.35(0.24-0.47)^{c^{*}}$ & $1.00(0.94-1.00)$ & $0.98(0.91-1.00)$ & $0.99(0.92-1.00)$ \\
\hline 5 & $0.20(0.11-0.32)$ & $0.14(0.06-0.26)^{b}$ & $0.32(0.21-0.44)^{c^{*}}$ & $0.98(0.92-1.00)$ & $0.97(0.88-1.00)$ & $0.93(0.84-0.98)$ \\
\hline $6 \mathrm{~A}$ & $0.39(0.27-0.52)$ & $0.38(0.25-0.51)^{b^{*}}$ & $0.75(0.63-0.85)^{c^{*}}$ & $0.98(0.92-1.00)$ & $0.98(0.91-1.00)$ & $1.00(0.95-1.00)$ \\
\hline 6B & $0.19(0.10-0.30)$ & $0.16(0.08-0.28)^{b^{*}}$ & $0.48(0.36-0.60)^{c^{*}}$ & $0.98(0.91-1.00)$ & $0.97(0.88-1.00)$ & $0.99(0.92-1.00)$ \\
\hline 7F & $0.64(0.51-0.76)$ & $0.68(0.54-0.80)^{b}$ & $0.86(0.75-0.93)^{c}$ & $0.98(0.92-1.00)$ & $1.00(0.94-1.00)$ & $1.00(0.95-1.00)$ \\
\hline 14 & $0.06(0.02-0.15)$ & $0.09(0.03-0.19)^{b^{*}}$ & $0.39(0.27-0.51)^{c^{*}}$ & $0.98(0.92-1.00)$ & $0.98(0.91-1.00)$ & $0.99(0.92-1.00)$ \\
\hline 18C & $0.86(0.75-0.93)$ & $0.95(0.85-0.99)$ & $0.99(0.92-1.00)^{c}$ & $1.00(0.94-1.00)$ & $1.00(0.94-1.00)$ & $1.00(0.95-1.00)$ \\
\hline $19 \mathrm{~A}$ & $0.06(0.02-0.15)$ & $0.09(0.03-0.20)^{b^{*}}$ & $0.35(0.24-0.47)^{c^{*}}$ & $1.00(0.94-1.00)$ & $0.97(0.88-1.00)$ & $0.94(0.86-0.98)$ \\
\hline 19F & $0.39(0.27-0.53)$ & $0.57(0.43-0.70)$ & $0.64(0.51-0.75)^{c}$ & $1.00(0.94-1.00)$ & $1.00(0.94-1.00)$ & $1.00(0.95-1.00)$ \\
\hline 23F & $0.63(0.50-0.75)$ & $0.49(0.35-0.63)^{b^{*}}$ & $0.78(0.67-0.87)$ & $1.00(0.94-1.00)$ & $1.00(0.94-1.00)$ & $1.00(0.95-1.00)$ \\
\hline
\end{tabular}


Figure 1: Consort diagram

Figure 2: Pneumococcal IgG GMCs following primary vaccination for each serotype and group. a b c: $p<0.05$ comparing groups 1 and 2, 2 and 3, and 1 and 3 respectively. Black capped lines indicate $95 \%$ confidence intervals, solid horizontal red line indicates $0.35 \mu \mathrm{g} / \mathrm{mL}$.

Figure 3: Pneumococcal IgG GMCs following booster vaccination for each serotype and group. a b c: $\mathrm{p}<0.05$ comparing groups 1 and 2, 2 and 3, and 1 and 3 respectively. Black capped lines indicate $95 \%$ confidence intervals, solid horizontal red line indicates $0.35 \mu \mathrm{g} / \mathrm{mL}$. 